\title{
Use of a Spacer Block Tool for Assessment of Joint Line Position during Revision Total Knee Arthroplasty
}

\author{
Harun R. Gungor, MD ${ }^{1}$ Nusret Ok, MD ${ }^{1}$ (이 \\ ${ }^{1}$ Department of Orthopedics and Traumatology, Faculty of Medicine, \\ Pamukkale University, Denizli, Turkey \\ J Knee Surg 2022;35:1260-1267.
}

Address for correspondence Harun R. Gungor, MD, Department of Orthopedics and Traumatology, Faculty of Medicine, Pamukkale University, 20070 Kinikli, Denizli, Turkey (e-mail: hrgungor@gmail.com).

\begin{abstract}
Keywords

- revision total knee arthroplasty

- joint line

- spacer block

- fibular head

- medial epicondyle
\end{abstract}

There is a tendency of orthopaedic surgeons to elevate joint line (JL) in revision total knee arthroplasty (RTKA). Here, we ascertain the use of the spacer block tool (SBT) to determine JL more accurately for less experienced RTKA surgeons. To perform more precise restoration of JL, an SBT with markers was developed and produced using computer software and three-dimensional printers. The study was planned prospectively to include patients who received either condylar constrained or rotating hinge RTKA between January 2016 and December 2019. To determine JL, distance from fibular head (FH), adductor tubercle (AT), and medial epicondyle (ME) were measured on contralateral knee preoperative radiographs and on operated knee postoperative radiographs. Patients were randomized and grouped according to the technique of $\mathrm{JL}$ reconstruction. In Group 1, conventional methods by evaluating aforementioned landmarks and preoperative contralateral knee measurements were used to determine $\mathrm{JL}$, whereas in Group 2, the SBT was used. The main outcome measure was the JL change in revised knee postoperatively in contrast to contralateral knee to compare effective restoration of JL between the groups. Twenty-five patients in Group 1 ( 3 males, 22 females, 72 years, body mass index [BMI] $32.04 \pm 4.45$ ) and 20 patients ( 7 males, 13 females, 74 years, BMI $30.12 \pm 5.02$ ) in Group 2 were included in the study. JL measurements for the whole group were $\mathrm{FH}-\mathrm{JL}=18.3 \pm 3.8 \mathrm{~mm}$, AT- $\mathrm{JL}=45.8 \pm 4.6$ $\mathrm{mm}$, and $\mathrm{ME}-\mathrm{JL}=27.1 \pm 2.8 \mathrm{~mm}$ preoperatively, and $\mathrm{FH}-\mathrm{JL}=20.7 \pm 4.2 \mathrm{~mm}$, AT-JL $=43.4 \pm 5.2 \mathrm{~mm}$, and $\mathrm{ME}-\mathrm{JL}=24.7 \pm 3.1 \mathrm{~mm}$ postoperatively. JL level differences in reference to $\mathrm{FH}, \mathrm{AT}$, and $\mathrm{ME}$ in Group 1 were $3.6 \pm 3.1,3.6 \pm 3.5$, and $3.4 \pm 3.1 \mathrm{~mm}$, respectively, and in Group 2 were $1.0 \pm .0 .9,1.3 \pm 1.3$, and $1.1 \pm 1.3 \mathrm{~mm}$, respectively. There were statistically significant differences between the two groups in JL changes referenced to all of the specific landmarks $(p<0.05)$. The use of the SBT helped restore $J L$ effectively in our cohort of RTKA patients. Therefore, this tool may become a useful and inexpensive gadget for less experienced and low-volume RTKA surgeons.
Assessment of natural joint line (JL) position during revision total knee arthroplasty (RTKA) is a challenging problem, and appropriate restoration of JL is critically important to achieve better clinical and functional outcomes. ${ }^{1-9}$ Although 2 to $5 \mathrm{~mm}$ of JL elevation has been reported in the literature as being not

received

April 25, 2020

accepted after revision

November 29, 2020

published online

January 20, 2021

associated with poor functional outcomes in primary TKA, ${ }^{10,11}$ extensor mechanism insufficiency, anterior knee pain, flexionextension imbalance, and loss of postoperative range of motion have been shown to be associated with JL elevation of more than 4 to $8 \mathrm{~mm}$ in revision cases. ${ }^{1,2,12,13}$ In addition, elevation of JL

(c) 2021. Thieme. All rights reserved. Thieme Medical Publishers, Inc., 333 Seventh Avenue, 18th Floor, New York, NY 10001, USA
DOI https://doi.org/ 10.1055/s-0040-1722628. ISSN $1538-8506$. 
results in pseudo patella baja condition where the patellar tendon length remains the same. ${ }^{1,3,4}$ This condition has been shown to produce significant changes in patellar kinematics and inferior edge loading between the patella and tibial components in biomechanical studies. ${ }^{1,3,4,14} \mathrm{JL}$ elevation along with tibial slope has also been reported to increase polyethylene wear in primary TKA. $^{6}$

There have been plenty of research studying appropriate restoration of JL in RTKA, and several techniques have been reported in the literature to adequately adjust JL level. ${ }^{3,15-17}$ Identification of meniscal remnants to use as a reference tool, detection of distance from adductor tubercle (AT) or from medial epicondyle (ME) to JL, and ratio of interepicondylar distance are all suggested techniques for restoration of JL in RTKA. ${ }^{15-27}$ Identification of meniscal remnants is not always possible in revision cases, and most commonly encountered error using femoral landmarks as a reference is JL proximalization resulting from inaccurate determination of aforementioned landmarks due to bone loss, individual variations of distal femoral anatomy, or technical errors. ${ }^{1-17}$ In addition, approximate values are used to detect JL in most of these techniques. ${ }^{19-27}$ Hence, it has been reported in the literature that there is a tendency of surgeons to proximalize and elevate $\mathrm{JL} \sim 5$ to $8 \mathrm{~mm}$ in revision cases. ${ }^{1,15,17}$

Approximate values for determination of JL referencing tibial landmarks namely fibular head (FH) and tibial tuberosity have not been validated in the literature. ${ }^{28,29}$ Tibial referencing should be more desirable for determination of JL if appropriate tibial landmark is identified and exact values for JL restoration are calculated. Assessment of JL by means of radiographs of contralateral knee has also been postulated, and the distance from the FH to JL in these radiographs found to be the most reliable parameter. ${ }^{30,31}$ If the appropriate JL position determined relative to $\mathrm{FH}$ in the contralateral knee, tibial surface is re-established first, followed by femoral sizing along with determination of distal and posterior augment blocks to set femoral component by evaluating AT, ME, FH, and flexion and extension gaps. However, intraoperative exploration of the tip of FH, ME, or AT to measure distance to JL usually exhibits some difficulties for less experienced RTKA surgeons during the procedure and fluoroscopic assessment needs to be utilized. For these circumstances, we developed a spacer block tool (SBT) with markers by using computer software and threedimensional (3D) printers to help determination of JL level during fluoroscopic evaluation of the knee joint for lowvolume RTKA surgeons. Here, we ascertain the use of this tool, and we also proposed that this tool also shortens operative time by eliminating some steps during the procedure.

\section{Materials and Methods}

This study was conducted prospectively in randomized manner at the department of orthopedics and traumatology in our university hospital. The study was approved by the Clinical Research and Ethics Committee of the authors' affiliated institution and conducted in accordance with the principles set forth in the Declaration of Helsinki 2008 (No: 60116787-020/8819). Informed consent was obtained from all participants.
Inclusion criteria were as follows: patients scheduled for one-stage RTKA surgery due to aseptic loosening, implant failure, and instability; or for a second-stage spacer exchange RTKA surgery. Exclusion criteria were as follows: RTKA surgery necessitating the use of segmental prosthesis, previous major orthopaedic surgery in either lower extremities deteriorating alignment of the extremity or compromising determination of JL on the contralateral knee. Patients with well-functioning primary knee arthroplasty (without an evidence of loosening, laxity, or malposition compromising determination of JL) on contralateral knee were not excluded.

Patients were randomized into two groups by a computer program according to the technique of JL reconstruction. All the operations were performed by the same surgeon using either condylar constrained RTKA (NexGen Legacy Condylar Constrained Knee, Zimmer-Biomet Inc., Warsaw, IN), or rotating hinge RTKA (NexGen Rotating Hinge Knee, Zimmer-Biomet Inc.). Medial parapatellar approach was used in all cases. In Group 1, conventional methods by evaluating specific landmarks intraoperatively and preoperative JL measurements from contralateral knee were used to determine revised knee JL, whereas in Group 2, the SBT was used. High-viscosity polymethyl methacrylate bone cement (Oliga-G21 srl-Vias. Pertini, San Possodonio, MO, Italy) was used for all patients.

\section{Measurements}

Long-leg radiographs of the patients were evaluated pre- and postoperatively by using a computer-based Digital Imaging and Communications in Medicine system (Probel, 2.0.9.0) and by using the same computer terminal with a highresolution monitor. The main outcome measure was the JL change in revised knee postoperatively in contrast to contralateral knee to compare effective restoration of JL between the two groups. Therefore, to assess JL in contralateral knee preoperatively and in revised knee postoperatively, three landmarks, namely AT, ME, and tip of the FH, were determined on long-leg standing radiographs. Distance from AT to JL (AT-JL), from ME to JL (ME-JL), and from the tip of the FH to JL (FH-JL) were measured and recorded. All the measurements were performed by the same observer blind to the group of the patients. For intrarater reliability test, measurements were repeated in two successive sessions with at least 2-hour intervals. The first measurements were used for evaluations.

\section{Operative Technique and the Spacer Block Tool to Determine Joint Line}

The principles of tibia-first gap balancing technique during RTKA are utilized in this method to assess JL position. Preoperatively, all the measurements were performed on contralateral knee to determine original JL level. Following determination of JL level relative to tip of the FH, AT, and ME on the radiographs of contralateral knee, more precise verification and easier configuration of tibial and femoral components are the aims of this proposal.

In Group 1, following tibial surface cut, the measurements of FH-JL from contralateral knee were used to determine 


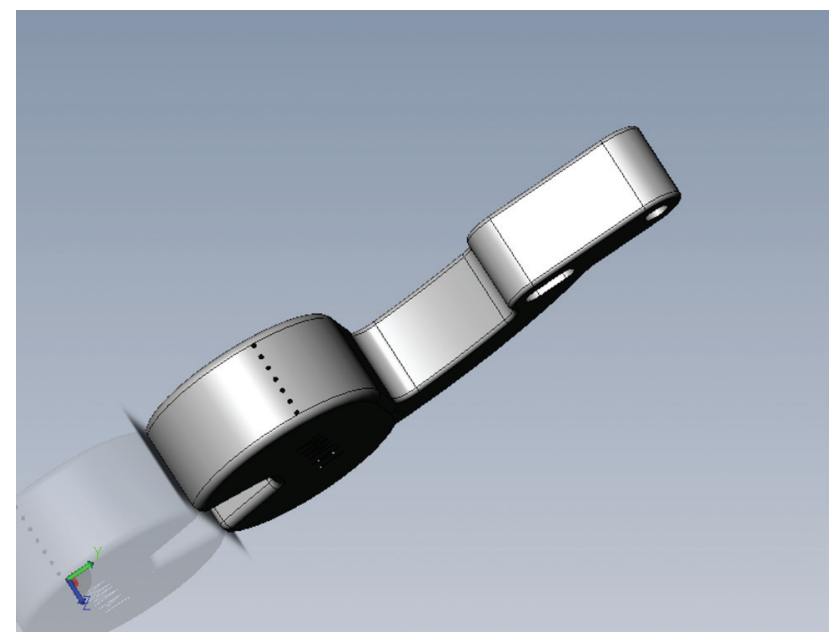

Fig. 1 Three dimensional (3D) model. 3D model drawn with SolidWorks software.

proper JL position intraoperatively by palpating and identifying $\mathrm{FH}$ in operated knees. Appropriate tibial augment and/or block, stem, and insert trial components were built and inserted to tibia. Following insertion of these tibial trial components, femoral distal, anterior, and chamfer cuts were performed, respectively, and size of femoral component, distal, anterior, and posterior augment blocks were deter- mined to establish predetermined JL level. At this step, preoperatively measured contralateral knee ME-JL and/or AT-JL values were also used to double-checked to assess newly structured JL level. Following insertion of the femoral trial components, the total revision arthroplasty system was tested and verified fluoroscopically. If desired soft tissue tension could not be restored with condylar constrained revision system while testing the trial components, rotating hinge revision system with the same augments and/or blocks were used to maintain previously measured and decided JL level. Following verification of trial components, permanent original components were prepared and inserted with the use of bone cement.

In Group 2, the surgeon made use of the SBT to determine JL level. The new form of SBT used in these operations was designed by using SolidWorks software (Dassault Systèmes, Paris, France) and produced by using CubeX 3D printers (3D Systems Inc., South Carolina) (-Fig. 1).

The SBT contains markers for measurements during fluoroscopic evaluation of JL. This SBP printed in 5 thicknesses starting from $21 \mathrm{~mm}$ with $4 \mathrm{~mm}$ increments till $37 \mathrm{~mm}$ (21, $25,29,33$, and $37 \mathrm{~mm}$ ). Two $\mathrm{mm}$ of clear spacer block part lies on each side and beginning from the third $\mathrm{mm}$, in every fourth $\mathrm{mm}$ of the spacer block in coronal plane, $1 \mathrm{~mm}$ of tubular space is located horizontally in printed sample. Following production of all samples, K-wires $1 \mathrm{~mm}$ in

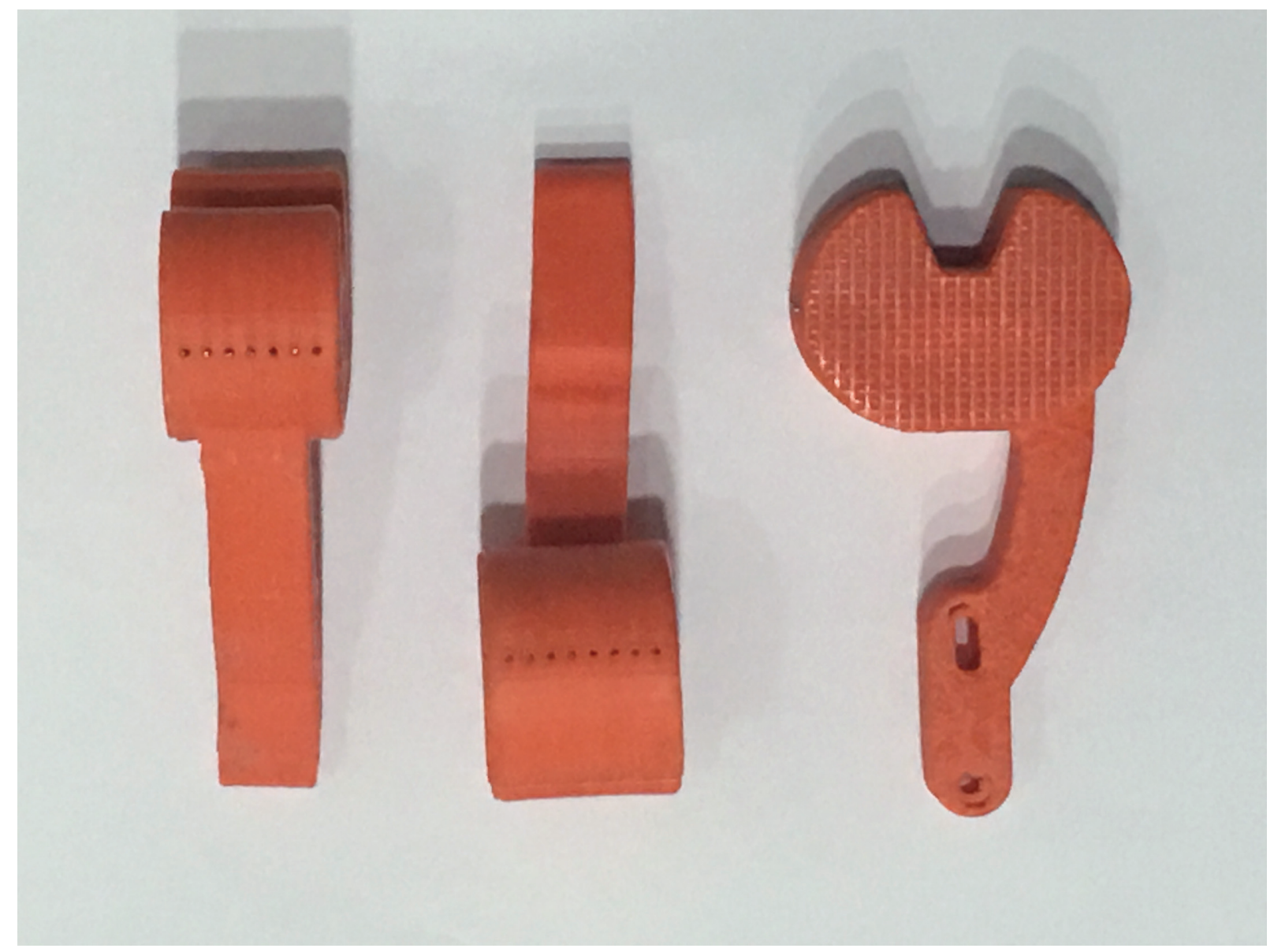

Fig. 2 Spacer block tool. Spacer block tool is marked with K-wires (1 mm thickness) traversing horizontally in coronal plane. Two mm of unmarked spacer block part lies on each end. K-wire markers are $1 \mathrm{~mm}$ width and clear space in between markers are $3 \mathrm{~mm}$. 


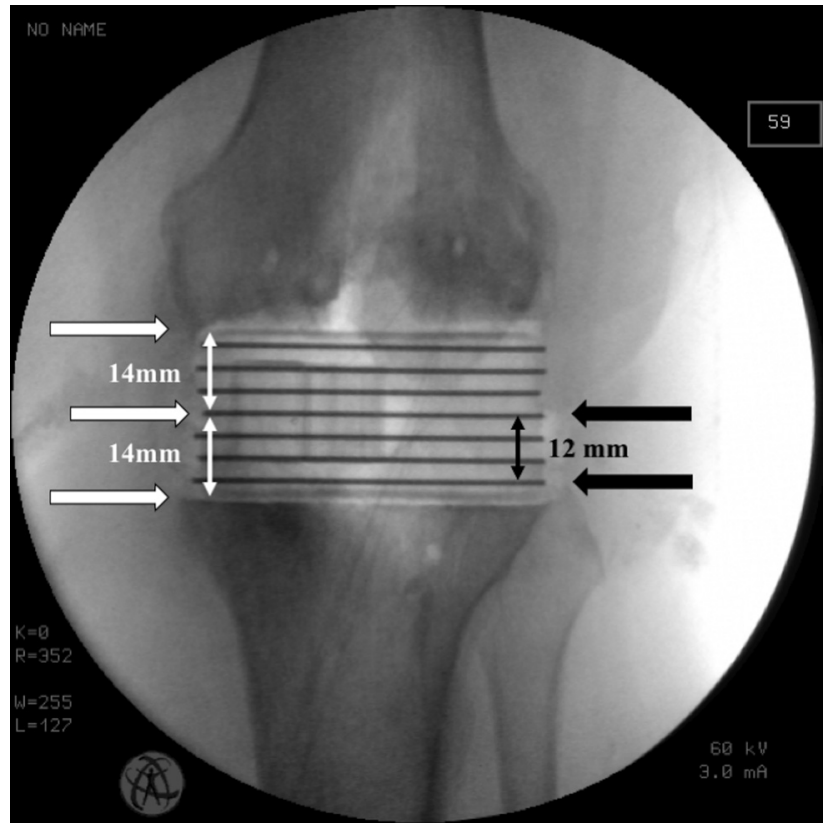

Fig. 3 Determination of JL. Preoperatively determined JL level in contralateral knee referencing tip of the fibular head was $12 \mathrm{~mm}$ in this revision TKA case. To balance extension gap, $29 \mathrm{~mm}$ novel spacer block tool was used. JL level was determined using markers for measurement (proximal black arrow) eliminating magnification errors since the distance between markers had already been known. Distance from JL marker to tibial cut surface was calculated as $14 \mathrm{~mm}$ (space between two distal white arrows). Distance from JL marker to distal femoral cut is $14 \mathrm{~mm}$. Therefore, extension gap should be balanced using $14 \mathrm{~mm}$ tibial construct and $14 \mathrm{~mm}$ distal femoral construct (total $28 \mathrm{~mm}$ ). Remaining $1 \mathrm{~mm}$ referring to the thickness of marker at determined JL may be ignored considering tibial and femoral cement mantle. JL, joint line; TKA, total knee arthroplasty. thicknesses were passed through these spaces as markers (-Fig. 2). Hence, $21 \mathrm{~mm}$ SBT contains five markers, $25 \mathrm{~mm}$ block contains six markers, and so on till $37 \mathrm{~mm}$ SBT which contains nine markers. The SBTs are ethylene oxide sterilized. Following proximal tibial and distal femoral cuts, ligaments are balanced (rectangular extension gap) and appropriate thickness SBT is inserted to check alignment of lower extremity and extension gap. Assuming that the alignment and ligament balance are adequate, fluoroscopic image of knee in anteroposterior plane is obtained (-Fig. 3). The markers allow easy detection of predetermined JL level measurement from contralateral knee referencing tip of the $\mathrm{FH}$, and also eliminate errors resulting from fluoroscopic imaging magnification of the extension gap. The thickness of SBT distal to marked JL corresponds to tibial construct thickness (polyethylene insert, tibial base plate, and if desired proximal tibial augment), and the thickness of SBT proximal to marked JL corresponds to distal femoral construct thickness (distal thickness of femoral component and thickness of distal femoral augment if needed). During fluoroscopic imaging, preoperatively measured contralateral knee ME-JL and/or AT-JL values were also used to double-check the JL level. At this step, appropriate tibial augment and/or block, stem, and insert trial components were built and inserted to tibia. This step was followed by determination of femoral component rotation, size, and posterior-anterior augments to establish flexion gap followed by femoral chamfer cuts. At this time, there is no need to re-establish extension gap since distal femoral construct predetermined during fluoroscopic assessment of JL to shorten duration of operation. Finally, femoral construct was built and trial components were inserted for reduction ( - Fig. 4). If desired soft tissue tension could not be restored with condylar constrained revision system while testing the trial components, rotating hinge

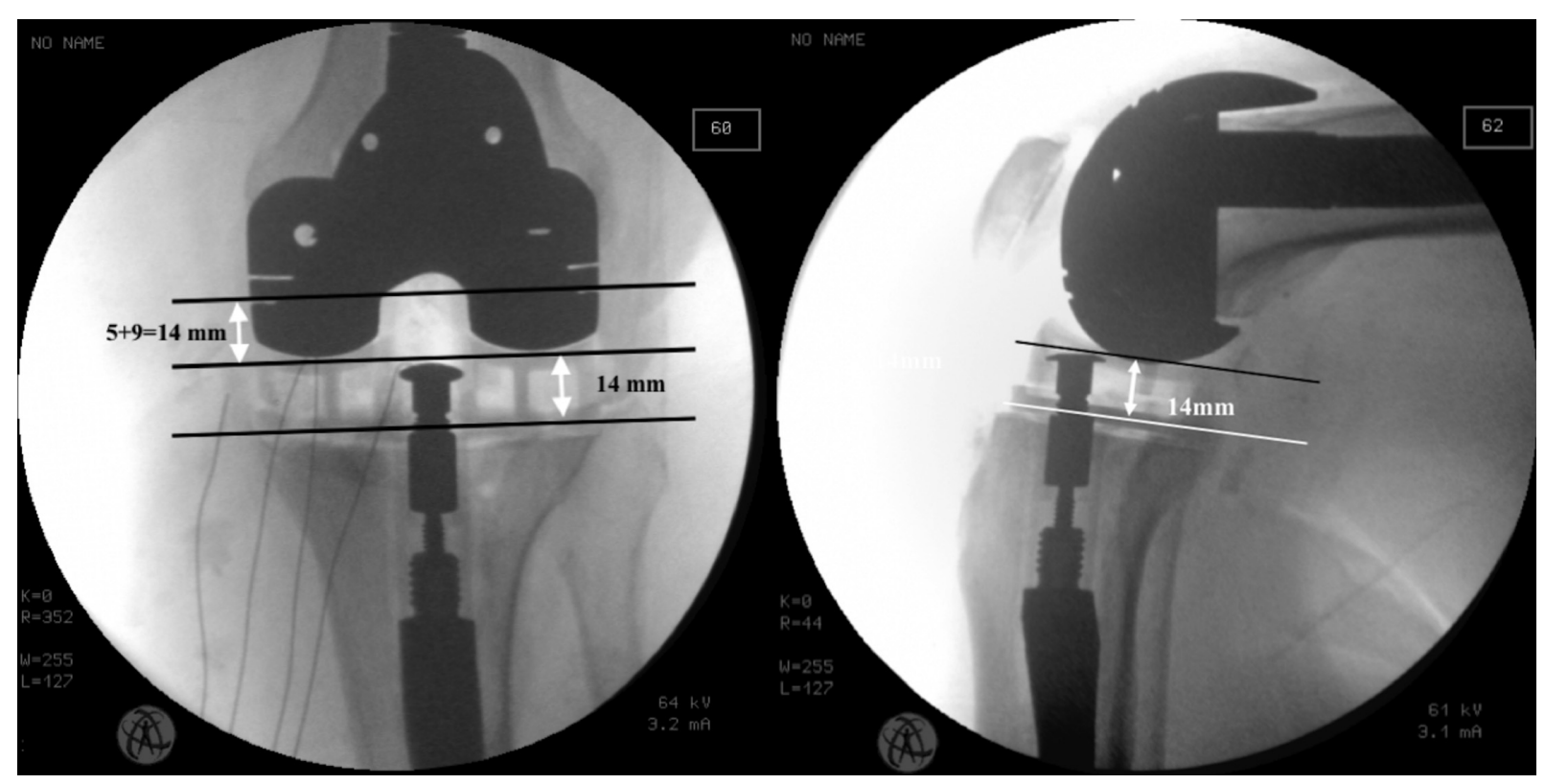

Fig. 4 Final construct. Femoral and tibial constructs were built and trial components were inserted. In this case, $14 \mathrm{~mm}$ tibial construct (base plate thickness included in the thickness of the insert in the arthroplasty instrument system used) and 5 mm distal medial and lateral femoral augment blocks $(5+9 \mathrm{~mm}$ [distal femoral component thickness] $=14 \mathrm{~mm}$ ) were used to balance extension gap. 
revision system with the same augments and/or blocks were used to maintain previously measured and decided JL level. Following verification of trial components, permanent original components were prepared and inserted with the use of bone cement.

Postoperatively same measurements (AT-JL, ME-JL, and FH-JL) were performed on revised knee radiographs to determine appropriate restoration of index JL measurements on the contralateral knee. Changes of JL levels postoperatively in contrast to preoperative measurements were compared between the two groups and statistical analyses were performed.

\section{Statistical Analysis}

An a priori power analysis (tested against a constant of 0.00 ) proved that at least 15 cases were required to detect a significant difference of JL elevation between groups with $95 \%$ power $(p<0.05, \alpha=0.05, \beta=0.05)$. An a posteriori power analysis (tested against a constant of 0.00 ) showed $97 \%$ power to detect a significant difference of JL elevation between groups with 20 cases $(p<0.05, \alpha=0.05, \beta=0.03$ ). The data were analyzed with the statistical package for social sciences software (SPSS version 17, Chicago, IL). Descriptive data are presented as mean \pm standard deviation or frequency (percentage). Intraclass correlation coefficient (ICC with $95 \%$ confidence interval) was used for intrarater reliability. Mean comparisons of the two groups were done using Mann-Whitney's $U$ test. Spearman's coefficient was used to test correlations between different measurements. Statistical significance was set at $p<0.05$.

\section{Results}

Forty-five patients were enrolled in this study from January 2016 to December 2019. Twenty-five patients in Group 1 ( 3 males, 22 females, mean age 72 years, mean body mass index [BMI] $32.04 \pm 4.45$ ) and 20 patients ( 7 males, 13 females, mean age 74 years, BMI $30.12 \pm 5.02$ ) in Group 2 were included in the study. Thirty-two patients ( 17 patients in Group 1 and 15 patients in Group 2) received condylar constrained RTKA and 13 patients ( 8 patients in Group 1 and 5 patients in Group 2) received rotating hinge RTKA.
Intrarater reliability tests were high for all the measurements ( - Table 1). ICCs were between 0.957 and 0.989 for all measurements $(p=0.0001)$.

JL measurements (mean \pm standard deviation [SD]) for the whole group were $\mathrm{FH}-\mathrm{JL}=18.3 \pm 3.8 \mathrm{~mm}$, AT-JL $=45.8$ $\pm 4.6 \mathrm{~mm}$, and $\mathrm{ME}-\mathrm{JL}=27.1 \pm 2.8 \mathrm{~mm}$ preoperatively, and $\mathrm{FH}-\mathrm{JL}=20.7 \pm 4.2 \mathrm{~mm}, \quad \mathrm{AT}-\mathrm{JL}=43.4 \pm 5.2 \mathrm{~mm}$, and $\mathrm{ME}-\mathrm{JL}$ $=24.7 \pm 3.1 \mathrm{~mm}$ postoperatively. JL measurements (mean \pm $\mathrm{SD}$ ) for Group 1 were $\mathrm{FH}-\mathrm{JL}=17.5 \pm 3.0 \mathrm{~mm}$, AT-JL $=44.9$ $\pm 4.1 \mathrm{~mm}$, and $\mathrm{ME}-\mathrm{JL}=26.9 \pm 3.4 \mathrm{~mm}$ preoperatively, and $\mathrm{FH}-\mathrm{JL}=21.0 \pm 3.9 \mathrm{~mm}, \quad \mathrm{AT}-\mathrm{JL}=41.3 \pm 4.5 \mathrm{~mm}$, and $\mathrm{ME}-\mathrm{JL}$ $=23.4 \pm 3.3 \mathrm{~mm}$ postoperatively. JL measurements (mean \pm $\mathrm{SD}$ ) for Group 2 were $\mathrm{FH}-\mathrm{JL}=19.2 \pm 4.4 \mathrm{~mm}$, AT-JL $=47.4$ $\pm 4.9 \mathrm{~mm}$, and $\mathrm{ME}-\mathrm{JL}=27.4 \pm 1.7 \mathrm{~mm}$ preoperatively, and $\mathrm{FH}-\mathrm{JL}=20.2 \pm 4.6 \mathrm{~mm}, \quad \mathrm{AT}-\mathrm{JL}=46.1 \pm 4.7 \mathrm{~mm}$, and $\mathrm{ME}-\mathrm{JL}$ $=26.2 \pm 2.0 \mathrm{~mm}$ postoperatively (-Table $\mathbf{2}$ ).

$\mathrm{JL}$ level differences (mean $\pm \mathrm{SD}$ ) in reference to $\mathrm{FH}, \mathrm{AT}$, and $\mathrm{ME}$ in Group 1 were $3.6 \pm 3.1,3.6 \pm 3.5$, and $3.4 \pm 3.1 \mathrm{~mm}$, respectively; and in Group 2 were $1.0 \pm 0.9,1.3 \pm 1.3$, and $1.1 \pm 1.3 \mathrm{~mm}$, respectively. There were statistically significant differences between the two groups in the JL changes referenced to all of the specific landmarks $(p<0.05)$ ( - Table 3 ).

\section{Discussion}

In the presented tibia-first gap balancing technique for RTKA, the SBT with markers allowed more reliable determination of JL level predetermined relative to tip of the $\mathrm{FH}$ on the radiographs of contralateral knee. Although there was a statistically significant difference between the groups, the clinical relevance of this difference was insignificant according to the literature; however, the SBT may become a useful guide for less experienced and low-volume RTKA surgeons. Utilization of this tool also helps shortening of operational time by allowing construction of extension gap femoral and tibial components at one step.

Partington et $\mathrm{al}^{5}$ studied 99 RTKA cases and reported statistically significant difference in clinical score of the patients with more than $8 \mathrm{~mm}$ of JL elevation; however, they did not detect any correlation between JL change and clinical scores of the patients. On the contrary, Figgie et $\mathrm{al}^{1}$ reported significant correlation between JL elevation and anterior knee pain, and range of motion of the patients. In a more recent

Table 1 Intraobserver reliabilities of joint line level measurements

\begin{tabular}{|l|l|l|l|}
\hline Measurements & ICC & Upper-lower boundaries & $p$-Value \\
\hline Preoperative FH-JL ${ }^{\mathrm{a}}$ & 0.985 & $0.972-0.992$ & 0.0001 \\
\hline Postoperative FH-JL & 0.988 & $0.977-0.993$ & 0.0001 \\
\hline Preoperative AT-JL ${ }^{\text {b }}$ & 0.985 & $0.974-0.992$ & 0.0001 \\
\hline Postoperative AT-JL & 0.989 & $0.980-0.994$ & 0.0001 \\
\hline Preoperative ME-JL ${ }^{\mathrm{N}}$ & 0.957 & $0.924-0.976$ & 0.0001 \\
\hline Postoperative ME-JL & 0.978 & $0.961-0.988$ & 0.0001 \\
\hline
\end{tabular}

Abbreviation: ICC, intraclass correlation coefficient.

${ }^{\mathrm{a}} \mathrm{FH}-\mathrm{JL}-$ measurement of distance from fibular head to joint line.

${ }^{\mathrm{b}} \mathrm{AT}-\mathrm{JL}-\mathrm{measurement}$ of distance from adductor tubercle to joint line.

${ }^{\mathrm{C}} \mathrm{ME}-\mathrm{JL}-$ measurement of distance from medial epicondyle to joint line. 
Table 2 Measurements for determination of joint line level

\begin{tabular}{|l|l|l|l|}
\hline Measurements $(\mathrm{mm})$ & Whole groups $(\boldsymbol{n}=\mathbf{4 5})$ & Group $\mathbf{1}(\boldsymbol{n}=\mathbf{2 5})$ & Group 2 ( $\boldsymbol{n}=\mathbf{2 0 )}$ \\
\cline { 2 - 4 } & $\begin{array}{l}\text { Mean } \pm \text { SD } \\
\text { (minimum-maximum) }\end{array}$ & $\begin{array}{l}\text { Mean } \pm \text { SD } \\
\text { (minimum-maximum) }\end{array}$ & $\begin{array}{l}\text { Mean } \pm \text { SD } \\
\text { (minimum-maximum) }\end{array}$ \\
\hline Preoperative FH-JL & $18.3 \pm 3.8(11.0-28.9)$ & $17.5 \pm 3.0(13.0-25.2)$ & $19.2 \pm 4.4(11.0-28.9)$ \\
\hline Postoperative FH-JL & $20.7 \pm 4.2(10.9-29.9)$ & $21.0 \pm 3.9(14.9-29.9)$ & $20.2 \pm 4.6(10.9-29.8)$ \\
\hline Preoperative AT-JL & $45.8 \pm 4.6(35.9-55.6)$ & $44.9 \pm 4.1(35.9-52.6)$ & $47.4 \pm 4.9(39-55.6)$ \\
\hline Postoperative AT-JL & $43.4 \pm 5.2(34.9-54.8)$ & $41.3 \pm 4.5(34.9-49.2)$ & $46.1 \pm 4.7(38.5-54.7)$ \\
\hline Preoperative ME-JL & $27.1 \pm 2.8(18.9-35.1)$ & $26.9 \pm 3.4(18.9-35.1)$ & $27.4 \pm 1.7(23.1-30.0)$ \\
\hline Postoperative ME-JL & $24.7 \pm 3.1(17.9-30.8)$ & $23.4 \pm 3.3(17.9-30.8)$ & $26.2 \pm 2.0(21.3-29.1)$ \\
\hline
\end{tabular}

Abbreviations: ICC, intraclass correlation coefficient; SD, standard deviation.

${ }^{\mathrm{a}} \mathrm{FH}-\mathrm{JL}-$ measurement of distance from fibular head to joint line.

${ }^{\mathrm{b}} \mathrm{AT}-\mathrm{JL}-$ measurement of distance from adductor tubercle to joint line.

${ }^{\mathrm{C}} \mathrm{ME}-\mathrm{JL}-$ measurement of distance from medial epicondyle to joint line.

Table 3 Joint line changes in reference to specific landmarks

\begin{tabular}{|c|c|c|c|}
\hline & Group $1(n=25)$ & Group $2(n=20)$ & \multirow[t]{2}{*}{$p$-Value } \\
\hline & $\begin{array}{l}\text { Mean } \pm \text { SD } \\
\text { (minimum-maximum) }\end{array}$ & $\begin{array}{l}\text { Mean } \pm S D \\
\text { (minimum-maximum) }\end{array}$ & \\
\hline Preoperative-postoperative $\mathrm{FH}^{-\mathrm{JL}^{\mathrm{a}}}$ difference & $3.6 \pm 3.1(-3.7-8.1)$ & $1.0 \pm .0 .9(-0.6-2.9)$ & 0.000 \\
\hline Preoperative-postoperative AT-JL ${ }^{\mathrm{b}}$ difference & $3.6 \pm 3.5(-5.2-8.5)$ & $1.3 \pm 1.3(-2.3-2.4)$ & 0.000 \\
\hline Preoperative-postoperative ME-JL ${ }^{\mathrm{C}}$ difference & $3.4 \pm 3.1(-4.1-8.4)$ & $1.1 \pm 1.3(-2.2-2.9)$ & 0.000 \\
\hline
\end{tabular}

Abbreviation: SD, standard deviation.

${ }^{\mathrm{a}} \mathrm{FH}-\mathrm{JL}-$ measurement of distance from fibular head to joint line.

${ }^{\mathrm{b}} \mathrm{AT}-\mathrm{JL}-$ measurement of distance from adductor tubercle to joint line.

${ }^{\mathrm{C}} \mathrm{ME}-\mathrm{JL}-$ measurement of distance from medial epicondyle to joint line.

study, Han et $\mathrm{al}^{2}$ studied 166 RTKA cases retrospectively and found more than $5 \mathrm{~mm}$ of JL elevation in $56 \%$ of cases. The authors reported that femoral JL position was the only significant factor that affected the change of ROM after RTKA. They did not find any statistically significant correlation between JL change and postoperative clinical scores. Kowalczewski et al ${ }^{32}$ tested six cadaveric specimens biomechanically to decide about the consequences of JL elevation. In this biomechanical study, the authors simulated active deep knee squats and passive flexion-extension cycles in the specimens and found only a small posterior shift (of $\sim 3 \mathrm{~mm}$ ) during squatting after $4 \mathrm{~mm}$ of JL elevation. As a result, the authors concluded that JL elevation by $4 \mathrm{~mm}$ in RTKA did not cause significant biomechanical changes during passive knee range of motion and squatting in the tibiofemoral joint. They also postulated that clinical problems following JL elevation would probably arise in the patellofemoral joint or would be caused by JL elevation of more than $4 \mathrm{~mm}$. Fornalski et al ${ }^{13}$ also studied cadaveric specimens biomechanically to test patellofemoral contact area, contact pressure, and kinematics following TKA with an anatomic JL and after 4 and $8 \mathrm{~mm}$ of JL elevation. The authors reported a significant increase in contact pressure only at 30 degrees of knee flexion with $8 \mathrm{~mm}$ of JL elevation and three of the six specimens showed inferior edge loading of the patella component following $8 \mathrm{~mm}$ of $\mathrm{JL}$ elevation at 120 degrees of knee flexion.
Although direct correlation of JL elevation and detrimental effects on clinical outcome has not been reported in the literature, excessive JL elevation of more than 4 to $8 \mathrm{~mm}$ should obviously be avoided to obtain delicate balance between osseous anatomy and surrounding soft tissues, and to abstain from both tibiofemoral and patellofemoral dysfunction. ${ }^{15}$ In our cases, JL level elevation in reference to $\mathrm{FH}, \mathrm{AT}$, and $\mathrm{ME}$ in Group 1 were $3.6 \pm 3.1,3.6 \pm 3.5$, and $3.4 \pm 3.1 \mathrm{~mm}$, respectively, and in Group 2 were $1.0 \pm 0.9$, $1.3 \pm 1.3$, and $1.1 \pm 1.3 \mathrm{~mm}$, respectively. According to the literature, clinical significance of the JL elevation of this amount will not be expected. ${ }^{15}$ Therefore, restoration of JL level predetermined relative to tip of the FH on the radiographs of contralateral knee resulted in successful restoration of postoperative JL in both groups in our study. Although there were statistically significant differences between the two groups in the JL changes referenced to all of the specific landmarks $(p<0.05)$, this will not be expected to result in any change in clinical outcome since the difference was small; however, the SBT can be expected to help easier and more precise restoration of $\mathrm{JL}$ if the surgeon is not experienced in revision cases. Otherwise, JL level predetermined relative to tip of the $\mathrm{FH}$ and double-checked with ME-JL and/or AT-JL measurements almost equally restores postoperative JL level in our cohort, if the surgeon experienced in RTKA cases. 
In conventional gap balancing technique in RTKA, Khakharia and Scuderi ${ }^{3}$ recommended the three-step technique in which tibial surface re-established first, followed by selection of appropriately sized femoral component to establish flexion gap, and finally setting of the femoral component to establish extension gap. In this technique, the last step impacts patellar height and distal JL by identifying AT, lateral epicondyle, and ME and using approximate values reported in the literature $(45,25$, and $30 \mathrm{~mm}$, respectively). At this step, most commonly encountered error using femoral landmarks as a reference is JL proximalization resulting from inaccurate determination of femoral landmarks due to femoral bone loss, individual variations of distal femoral anatomy, or technical errors. ${ }^{15-17}$ To balance extension gap, a thicker insert is used, and corresponding flexion gap is balanced by downsizing femoral component. This may result in poor clinical and functional outcomes by elevating JL. ${ }^{16}$ Therefore, JL position should be rechecked additionally either by exploring location of meniscal remnants or by fluoroscopic imaging, or both during identification of JL referencing femoral landmarks. Instead, as it is in our JL restoration technique, tibial referencing is more desirable at this point if appropriate landmark, namely, FH is identified and exact values from the contralateral knee is used to level JL. Mean values for FH-JL, AT-JL, and ME-JL measured in our study were $\mathrm{FH}-\mathrm{JL}=18.3 \pm 3.8 \mathrm{~mm}$, AT-JL $=45.8 \pm 4.6 \mathrm{~mm}$, and $\mathrm{ME}-\mathrm{JL}=27.1 \pm 2.8 \mathrm{~mm}$ in preoperative contralateral knee radiographs. These measurements were in parallel with approximate values reported in the literature. , $^{3,15-18,20-28}$

Maderbacher et $\mathrm{al}^{30}$ studied the distances between bony landmarks and the JL in radiographs of contralateral knee. They measured distances from ME, lateral epicondyle, FH, and AT to JL, and searched relationship of these measurements with sex, age, the level of arthritis, and the extend of misalignment. Regarding the femoral landmarks, the AT was the best parameter that could be identified by two observers; however, both observers could clearly identify the AT only in $47 \%$ of the cases. The authors attributed these finding to presence of osteophytes, individual anatomic variations, and malposition of the extremity during radiographic image acquisition. According to the results of their study, the most precise parameter was found to be the distance between the FH and the JL with $97 \%$ agreement of the observers. ${ }^{30}$ This is particularly important from the point that once the tibial joint surface is accurately set, distal femoral augmentation is constructed eliminating the step used in the femoral landmark referencing techniques to determine JL. If the novel spacer block with markers is used, utilizing fluoroscopic images, the tibial construct thickness and the distal femoral construct thickness are ascertained: the thickness of SBT distal to marked JL corresponds to tibial construct thickness (polyethylene insert, tibial base plate, and if desired proximal tibial augment), and the thickness of SBT proximal to marked JL corresponds to distal femoral construct thickness (distal thickness of femoral component and thickness of distal femoral augment if needed). Once the JL is established in extension, flexion gap may be balanced by adjusting rotation, sizing the femur, and adding posterior or anterior augments. Therefore, the procedure is completed in two steps for assessing JL level and sizing of the components.

One of the limitations of our study is that we did not include cases from low-volume surgeons to compare JL reconstruction. Another limitation of our study is that we did not include clinical outcome scores of the patients in statistical analysis of the results. This is because we primarily aimed to test the efficacy of the SBT to level of JL radiographically. Second, primary diagnosis for RTKA in our group of patients were too scattered to be able to stabilize confounding factors and the number of patients were relatively small to statistically analyze clinical outcome scores. Last limitation to be mentioned is that, some of the patients planned for revision might be applied TKA previously on contralateral knee. In these cases, distal femoral JL should be used to determine desired JL level as validated before by Han et al. ${ }^{2}$ In these cases, either AT or ME, or FH may be used to assess level of JL. If RTKA applied to contralateral knee previously, this technique to determine level of JL by using SBT is not applicable unless otherwise the surgeon is confident about the successful restoration of the $\mathrm{JL}$ in the prior revision operation of the contralateral knee. This time, AT can be used to estimate level of JL on contralateral knee due to the expected bone loss in revision cases as suggested by Yeh et al. ${ }^{26}$

\section{Conclusion}

The SBT with markers used in our study allows effective restoration of JL level predetermined relative to tip of the FH on the radiographs of contralateral knee and saves operational time by aiding construction of extension gap femoral and tibial components at one step in RTKA. Therefore, this tool may become a useful and inexpensive gadget for less experienced and low-volume RTKA surgeons.

\section{Note}

Informed consent was obtained from all individual participants included in the study.

\section{Ethical Approval}

The study has been performed in compliance with the Declaration of Helsinki and has been approved by Pamukkale University Clinical Research Ethics Committee (No: 60116787 020/8819).

\section{Funding}

None.

\section{Conflict of Interest}

None declared.

\section{References}

1 Figgie HE III, Goldberg VM, Heiple KG, Moller HS III, Gordon NH. The influence of tibial-patellofemoral location on function of the knee in patients with the posterior stabilized condylar knee prosthesis. J Bone Joint Surg Am 1986;68(07):1035-1040

2 Han HS, Yu CH, Shin N, Won S, Lee MC. Femoral joint line restoration is a major determinant of postoperative range of 
motion in revision total knee arthroplasty. Knee Surg Sports Traumatol Arthrosc 2019;27(07):2090-2095

3 Khakharia S, Scuderi GR. Restoration of the distal femur impacts patellar height in revision TKA. Clin Orthop Relat Res 2012;470 (01):205-210

4 Matz J, Lanting BA, Howard JL. Understanding the patellofemoral joint in total knee arthroplasty. Can J Surg 2019;62(01):57-65

5 Partington PF, Sawhney J, Rorabeck CH, Barrack RL, Moore J. Joint line restoration after revision total knee arthroplasty. Clin Orthop Relat Res 1999;(367):165-171

6 Pourzal R, Cip J, Rad E, et al. Joint line elevation and tibial slope are associated with increased polyethylene wear in cruciate-retaining total knee replacement. J Orthop Res 2020;38(07):1596-1606

7 Sachdeva S, Baker JF, Bauwens JE, et al. Can revision TKA patients achieve similar clinical functional improvement compared to primaries? J Knee Surg 2020;33(12):1219-1224

8 van Lieshout WAM, Duijnisveld BJ, Koenraadt KLM, Elmans LHGJ, Kerkhoffs GMMJ, van Geenen RCI. Adequate joint line restoration and good preliminary clinical outcomes after total knee arthroplasty using the Flexion First Balancer technique. Knee 2019;26(03):794-802

9 van Lieshout WAM, Valkering KP, Koenraadt KLM, van EttenJamaludin FS, Kerkhoffs GMMJ, van Geenen RCI. The negative effect of joint line elevation after total knee arthroplasty on outcome. Knee Surg Sports Traumatol Arthrosc 2019;27(05):1477-1486

10 Babazadeh S, Dowsey MM, Vasimalla MG, Stoney JD, Choong PFM. Gap balancing sacrifices joint-line maintenance to improve gap symmetry: 5-year follow-up of a randomized controlled trial. J Arthroplasty 2018;33(01):75-78

11 Minoda Y, Sugama R, Ohta Y, Ueyama H, Takemura S, Nakamura H. Joint line elevation is not associated with mid-flexion laxity in patients with varus osteoarthritis after total knee arthroplasty. Knee Surg Sports Traumatol Arthrosc 2020;28(10):3226-3231

12 Yoshii I, Whiteside LA, White SE, Milliano MT. Influence of prosthetic joint line position on knee kinematics and patellar position. J Arthroplasty 1991;6(02):169-177

13 Fornalski S, McGarry MH, Bui CN, Kim WC, Lee TQ. Biomechanical effects of joint line elevation in total knee arthroplasty. Clin Biomech (Bristol, Avon) 2012;27(08):824-829

14 van Duren BH, Pandit H, Pechon P, Hart A, Murray DW. The role of the patellar tendon angle and patellar flexion angle in the interpretation of sagittal plane kinematics of the knee after knee arthroplasty: a modelling analysis. Knee 2018;25(02):240-248

15 Bellemans J. Restoring the joint line in revision TKA: does it matter? Knee 2004;11(01):3-5

16 Dennis DA. A stepwise approach to revision total knee arthroplasty. J Arthroplasty 2007;22(4, suppl 1):32-38

17 Laskin RS. Joint line position restoration during revision total knee replacement. Clin Orthop Relat Res 2002;(404):169-171

18 Fan A, Xu T, Li X, et al. Using anatomical landmarks to calculate the normal joint line position in Chinese people: an observational study. J Orthop Surg Res 2018;13(01):261

19 Iacono F, Lo Presti M, Bruni D, et al. The adductor tubercle: a reliable landmark for analysing the level of the femorotibial joint line. Knee Surg Sports Traumatol Arthrosc 2013;21(12): 2725-2729

20 Iacono F, Raspugli GF, Bruni D, et al. The adductor tubercle as an important landmark to determine the joint line level in total knee arthroplasty: from radiographs to surgical theatre. Knee Surg Sports Traumatol Arthrosc 2014;22(12):3034-3038

21 Iacono F, Raspugli GF, Filardo G, et al. The adductor tubercle: an important landmark to determine the joint line level in revision total knee arthroplasty. Knee Surg Sports Traumatol Arthrosc 2016;24(10):3212-3217

22 Lutz B, Trubrich A, Kappe T, Reichel H, Bieger R. The epicondylar ratio can be reliably used on X-ray of the knee to determine the joint line. Arch Orthop Trauma Surg 2018;138(09):1287-1292

23 Ozkurt B, Sen T, Cankaya D, Kendir S, Basarır K, Tabak Y. The medial and lateral epicondyle as a reliable landmark for intraoperative joint line determination in revision knee arthroplasty. Bone Joint Res 2016;5(07):280-286

24 Pereira GC, von Kaeppler E, Alaia MJ, et al. Calculating the position of the joint line of the knee using anatomical landmarks. Orthopedics 2016;39(06):381-386

25 Romero J, Seifert B, Reinhardt O, Ziegler O, Kessler O. A useful radiologic method for preoperative joint-line determination in revision total knee arthroplasty. Clin Orthop Relat Res 2010;468 (05):1279-1283

26 Yeh KT, Chen IH, Wang CC, Wu WT, Liu KL, Peng CH. The adductor tubercle can be a radiographic landmark for joint line position determination: an anatomic-radiographic correlation study. J Orthop Surg Res 2019;14(01):189

27 Xiao J, Wang S, Chen W, Yang Y, Liu T, Zuo J. A study to assess the accuracy of adductor tubercle as a reliable landmark used to determine the joint line of the knee in a Chinese population. J Arthroplasty 2017;32(04):1351-1355

28 Servien E, Viskontas D, Giuffrè BM, Coolican MR, Parker DA. Reliability of bony landmarks for restoration of the joint line in revision knee arthroplasty. Knee Surg Sports Traumatol Arthrosc 2008;16(03):263-269

29 Havet E, Gabrion A, Leiber-Wackenheim F, Vernois J, Olory B, Mertl P. Radiological study of the knee joint line position measured from the fibular head and proximal tibial landmarks. Surg Radiol Anat 2007;29(04):285-289

30 Maderbacher G, Keshmiri A, Zeman F, Grifka J, Baier C. Assessing joint line positions by means of the contralateral knee: a new approach for planning knee revision surgery? Knee Surg Sports Traumatol Arthrosc 2015;23(11):3244-3250

31 Clavé A, Le Henaff G, Roger T, Maisongrosse P, Mabit C, Dubrana F. Joint line level in revision total knee replacement: assessment and functional results with an average of seven years follow-up. Int Orthop 2016;40(08):1655-1662

32 Kowalczewski JB, Labey L, Chevalier Y, Okon T, Innocenti B, Bellemans J. Does joint line elevation after revision knee arthroplasty affect tibio-femoral kinematics, contact pressure or collateral ligament lengths? An in vitro analysis. Arch Med Sci 2015;11 (02):311-318 